\title{
Clinicopathological features of patients with transformation from EGFR mutant lung adenocarcinoma to small cell lung cancer
}

\author{
Wenwen Bai^, Chanjun Zhen, Ruohui Zhang, Wenhui Yu, Zhiguo Zhou^ \\ Department of Radiation Oncology, the Fourth Hospital of Hebei Medical University, Shijiazhuang, China \\ Contributions: (I) Conception and design: Z Zhou, W Bai; (II) Administrative support: Z Zhou; (III) Provision of study materials or patients: W Bai, \\ Z Zhou, C Zhen; (IV) Collection and assembly of data: W Bai, R Zhang, W Yu; (V) Data analysis and interpretation: W Bai, C Zhen, Z Zhou; (VI) \\ Manuscript writing: All authors; (VII) Final approval of manuscript: All authors. \\ Correspondence to: Zhiguo Zhou. Department of Radiation Oncology, the Fourth Hospital of Hebei Medical University, No. 12 Jiankang Road, \\ Shijiazhuang 050011, China. Email: chenk777@126.com.
}

\begin{abstract}
Background: Recently, an increasing number of cases with transformation from lung adenocarcinoma to small cell lung cancer (SCLC) have been identified, but few studies have investigated the clinical, pathological as well as molecular characteristics of these cases. This study aimed to summarize and analyze these features.
\end{abstract}

Methods: We retrospectively collected data including clinical information, laboratory examination results, radiological and pathological findings of ten patients, who were confirmed to undergo SCLC transformation following treatment from January 2014 to January 2020.

Results: The median time of treatment (targeted agents) was 14 months, and the median time interval of SCLC transformation following treatment was 24 months. Immunohistochemical indicators after transformation showed positive thyroid transcription factor 1 (TTF1), synaptophysin (Syn), CD56, and AE1/AE3, highly expressed Ki67, as well as negative programmed cell death-ligand 1 (PD-L1). Compared with the patients who received targeted therapy first, those patients who received chemotherapy followed by targeted therapy presented longer time intervals to transformation (36 vs. 22 months). Genetic testing after transformation showed that eight patients still maintained the original epidermal growth factor receptor (EGFR) mutation types. The median progression-free survival (PFS) after transformation was 5 months, and the median survival time after transformation was 10 months in seven patients who died.

Conclusions: Lung adenocarcinomas, once transformed to SCLC, progress rapidly and lead to poorer prognosis. After transformation, most of the patients maintain the original EGFR mutation types.

Keywords: Lung cancer; adenocarcinoma; small cell carcinoma; transformation; prognosis

Submitted Apr 15, 2021. Accepted for publication Jun 25, 2021.

doi: $10.21037 /$ tcr-21-653

View this article at: https://dx.doi.org/10.21037/tcr-21-653

\section{Introduction}

Currently, lung cancer is the most common cancer in the world, demonstrating the highest morbidity and mortality (1). Among them, non-small cell lung cancer (NSCLC) accounts for $80-85 \%$ of the total cases. Adenocarcinoma accounts for more than half of cases in terms of histological type. With the rapid development of precision medicine, molecular targeted drugs, such as epidermal growth factor receptor-tyrosine kinase inhibitor (EGFR-TKI), have achieved encouraging outcomes in patients with advanced NSCLC. However, most patients inevitably developed acquired resistance

^ ORCID: Wenwen Bai, 0000-0002-7037-648X; Zhiguo Zhou, 0000-0002-5373-8098. 

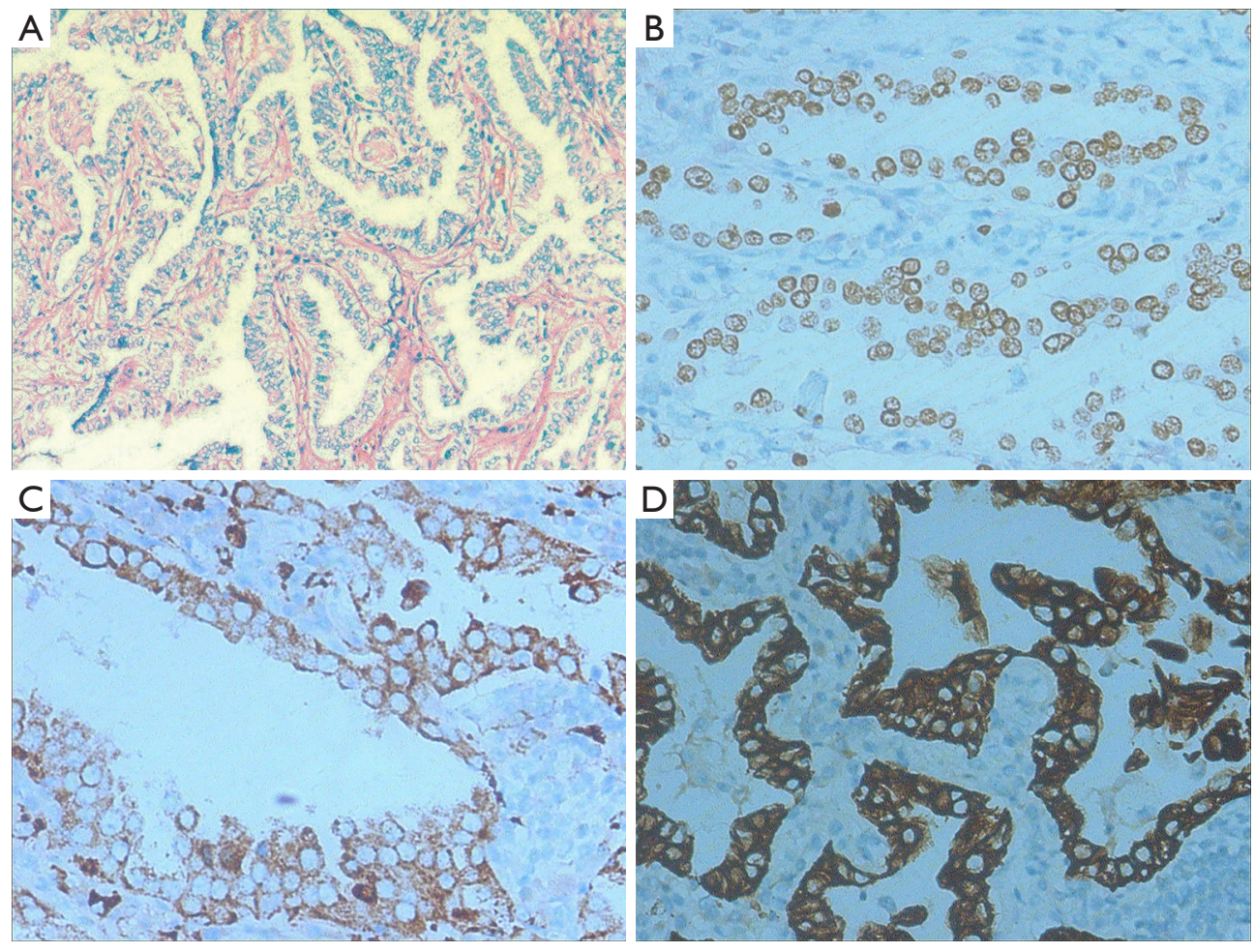

Figure 1 Pathological and immunohistochemical examination of case 1 at initial diagnosis (adenocarcinoma). (A) The initial pathology (H\&E, ×10). (B) TTF-1 positive (IHC, ×20). (C) Napsin A positive (IHC, ×20). (D) CK positive (IHC, ×20). H\&E, hematoxylin-eosin staining; IHC, immunohistochemical staining; TTF-1, thyroid transcription factor 1; CK, cytokeratin.

at 10-12 months after treatment (2). At present, the most common resistance mechanism is the T790M mutation, accounting for approximately $60 \%$. Other causes of resistance include c-Met gene amplification, human epidermal growth factor receptor 2 (HER2) gene amplification and the transformation of small cell lung cancer (SCLC) (3). Among them, the transformation of NSCLC to SCLC has brought much confusion to clinical diagnosis and treatment. Therefore, we should not ignore this unique phenomenon because of the low incidence. In 2006, Zakowski et al. first reported a NSCLC patient who was diagnosed with SCLC accompanied by the EGFR exon 19 mutation after TKI treatment (4). However, this patient had not undergone EGFR genetic test before the treatment. Subsequently, an increasing number of cases have been reported to exhibit such phenomenon (5-7), but few retrospective studies are currently able to analyze these cases meticulously and comprehensively. This study aimed to summarize and analyze the clinicopathological features of the patients with the transformation from lung adenocarcinoma into SCLC, thus providing a reference for future practice in clinic.

We present the following article in accordance with the STROBE reporting checklist (available at https://dx.doi. org/10.21037/tcr-21-653).

\section{Methods}

\section{Case data}

We collected the clinical and pathological data of ten patients who developed the transformation from lung adenocarcinoma to SCLC (mixed pathological types were excluded) following treatment from January 2014 to January 2020 in the Fourth Hospital of Hebei Medical University. Among them, seven cases were males and three were females. The average age was 55 (ranged from 30 to 68) years. All the patients were initially diagnosed with adenocarcinoma based on the pathological examination. However, only two cases underwent immunohistochemical examination (the pathological and immunohistochemical findings of case 1 at the first 
diagnosis were shown on Figure 1). Before transformation, three patients underwent resection of lower lobe of left lung with postoperative pathological examination, whereas the remaining seven patients underwent biopsies of lung masses. While all patients underwent needle biopsies and immunohistochemical examination to confirm the pathological diagnosis of SCLC after transformation. The neuron-specific enolase (NSE) values were 12.03, 14.76, $23.42,15.26,12.34,10.51,14.29,11.83,11.83,12.62 \mu \mathrm{g} / \mathrm{L}$ (reference value range: $0-16.3 \mathrm{ng} / \mathrm{L}$ ) in the included patients, and slightly elevated in one patient. The remaining information is summarized in Table 1. The study was conducted in accordance with the Declaration of Helsinki (as revised in 2013). The study was approved by the Ethics Committee board of the Fourth Hospital of Hebei Medical University (2020KY254) and individual consent for this retrospective analysis was waived.

\section{Study design}

We collected data including clinical information, laboratory examination results, radiological and pathological findings among the ten patients. Specifically, a retrospective review of the patients' medical records was performed focusing on the levels of tumor markers before transformation, the time point of SCLC transformation, gene mutation status before and after transformation, as well as the progression-free survival (PFS) and overall survival (OS) after transformation. The specific treatment for the 10 patients before transformation is displayed in Table 2. Genotyping was performed with next-generation sequencing (NGS). Besides, the pathological diagnosis was made by routine histopathological sections, which were stained with a Vantana automatic immunohistochemical staining instrument. The proportion of cells with positive programmed cell death-ligand 1 (PD-L1) expression was obtained by DAKO 22C3 pharmDx and VENTANA SP263.

\section{Follow-up}

The cutoff date for follow-up was August 1, 2020.

\section{Statistical analysis}

Statistical analysis was performed using SPSS 22.0 software. Descriptive statistics were presented as mean (or median) and range.

\section{Results}

\section{Clinical characteristics}

EGFR gene mutations were detected before the initial diagnosis in 9 of 10 patients, including 8 patients with EGFR exon 19 del (EGFR E19) mutation and 1 patient with EGFR exon 21 del L858R (EGFR E21 L858R) mutation based on their blood specimens. A caveat to this is that no gene mutation was found in case 10 at the initial diagnosis, therefore, TKI therapy was not provided. The median time of TKI treatment for the nine patients with EGFR mutation prior to SCLC transformation was 14 months (range, 7-21 months). Four patients (40\%) had the same site of neoplastic transformation as the primary, and tissue biopsies were performed in six patients to confirm transformation in the metastases $(60 \%)$. The images before and after transformation for case 4 are shown in Figure 2. In addition, NSE levels in 10 patients were all elevated before transformation (100\%), and the fold elevation was 1.1 to 8.2 , with an average elevation of $4.1(3.5 \pm 2.8)$. Disease progression occurs primarily at multiple sites prior to transformation, including the lung, bone, brain, pleura, and lymph nodes. Eight patients maintained the original EGFR gene mutation type (80\%) after transformation, but the mutation type of one patient changed to EGFR T790M mutation. Notably, the patient in case 10 with no EGFR mutation before transformation presented an EGFR E19 mutation after transformation. Relevant information for all patients is detailed in Table 1 .

\section{Pathological immunobistochemistry}

Immunohistochemistry after transformation showed positive thyroid transcription factor 1 (TTF1), synaptophysin (Syn), CD56, AE1/AE3, negative Napsin A, highly expressed Ki67, and 0\% expressed PD-L1 in all patients (Table 3, Figure 3). In addition, specimens of 5 cases were negative for chromogranin $\mathrm{A}(\mathrm{CgA})$.

\section{The time interval of transformation}

The time intervals between treatment and transformation from lung adenocarcinoma to SCLC were 7-40 months (median 24 months) in the 10 patients. In particular, the time interval to transformation for case 10 who did not receive targeted therapy was 34 months. The remaining 9 patients received targeted therapy before transformation. In terms of treatment sequence, 3 patients (cases 1, 2 and 8) 


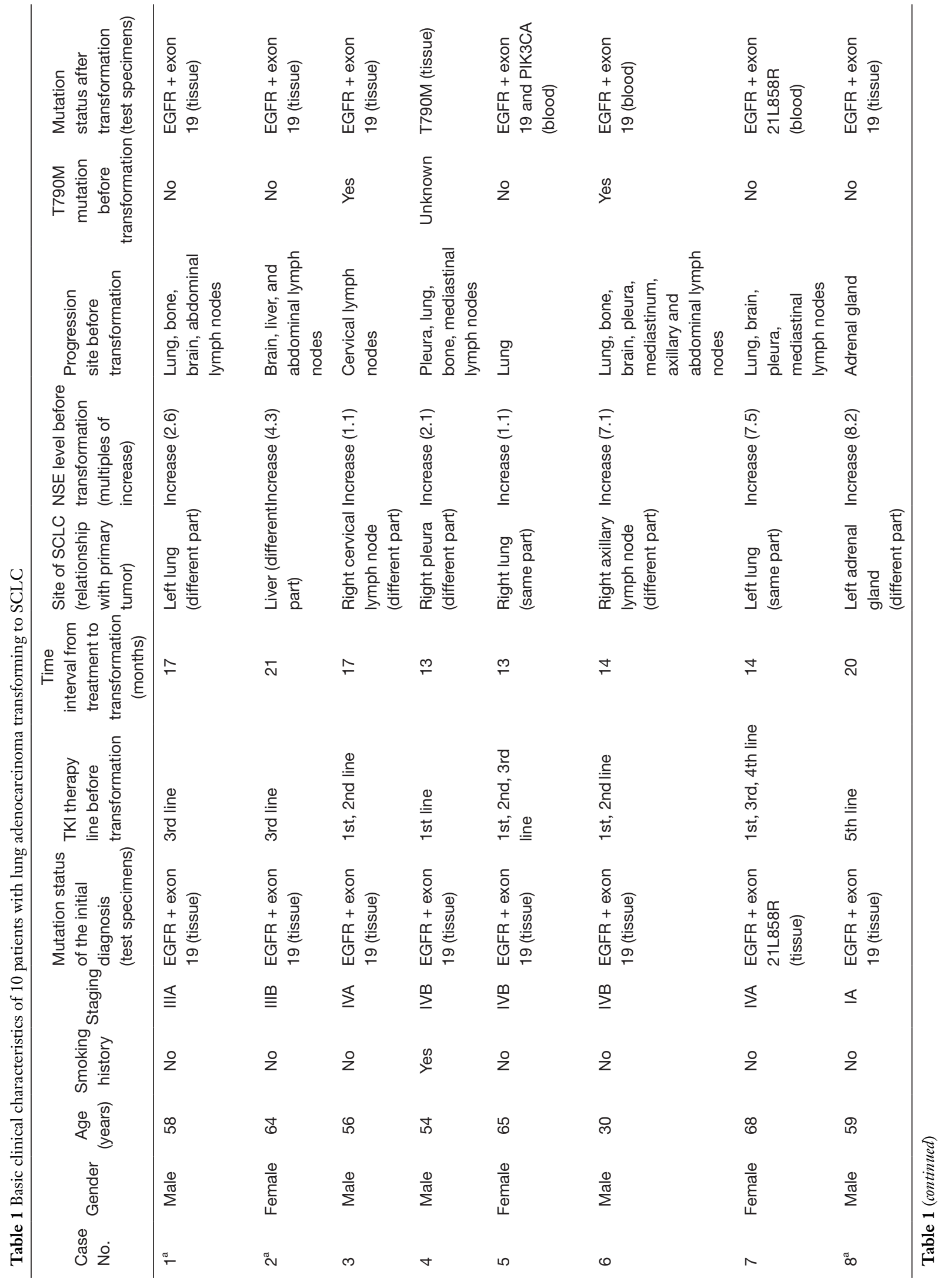




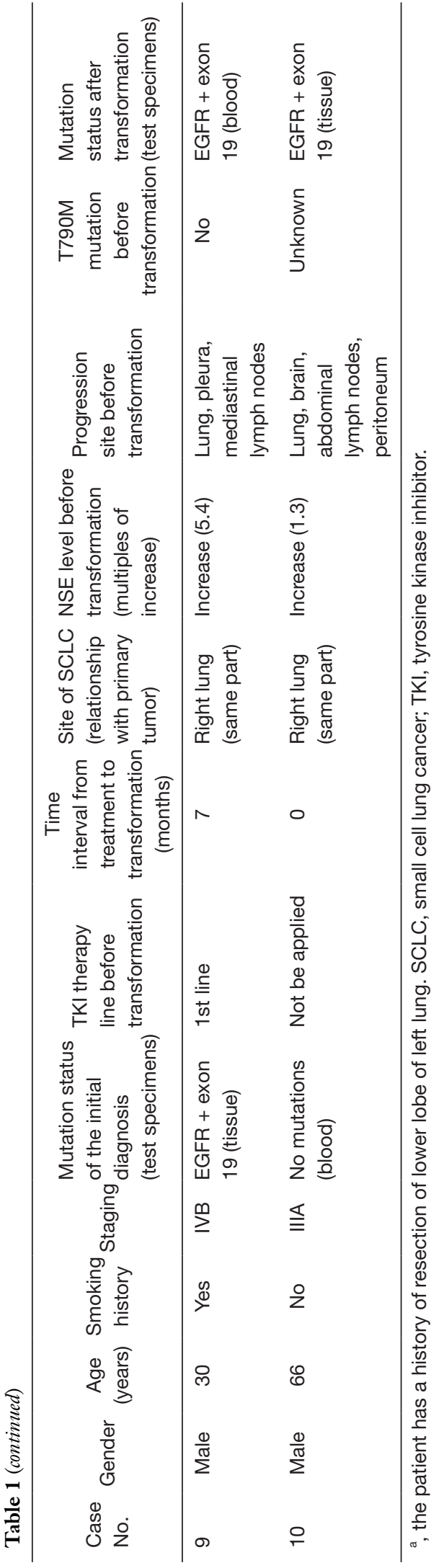

were treated with chemotherapy followed by targeted therapy $(\mathrm{C}+\mathrm{T})$ and their time intervals to transformation were 38, 40 and 31 months (mean 36 months), respectively. The group treated with targeted therapy followed by chemotherapy $(T+C)$ included cases 4,5 and 7 , whose time intervals to transformation were 13,14 , and 40 months (mean 22 months), respectively. In addition, 2 patients (cases 6 and 9) received concomitant chemotherapy combined with targeted therapy (CCT), and their transformation intervals were 15 and 7 months (mean 11 months), respectively. Besides, one patient (case 3) underwent targeted therapy alone and his transformation time was 17 months (Table 4). In brief, the $\mathrm{C}$ $+\mathrm{T}$ group demonstrated longer interval of transformation.

\section{The treatment and prognosis after transformation}

The therapeutic methods after transformation were mainly chemotherapy-based comprehensive treatment. As of August 1, 2020, seven patients had died, including two (cases 6 and 7) from brain metastases, four (cases 2, 4, 5 and 8) from liver metastases and one (case 10) from respiratory failure due to multiple lung metastases. For the seven patients who have died, the survival time after transformation was 6-13 months (median 10 months). PFS for all patients after transformation was 1-12 months (median 5 months after SCLC diagnosis) (Table 5).

\section{Discussion}

After receiving first-line EGFR-TKI treatment, the majority of advanced NSCLC patients with sensitive mutations in the EGFR gene would develop disease progression after 10 to 12 months, i.e., acquired resistance (2). The resistant phenotypes in $4 \%$ to $14 \%$ of patients are due to SCLC transformation (8). However, there is currently no clear consensus on the definition of transformation from lung adenocarcinoma into SCLC, and the mechanisms of drug resistance caused by SCLC transformation remain obscure. The prevailing view is that there may be three related mechanisms: (I) hypothesis of tumor cell heterogeneity: in the initial process of tumorigenesis, there are two mixed components in tumor tissue. SCLC and adenocarcinoma cells coexist in the same or different sites. The adenocarcinoma cells are the dominant component at initial period, under treatment EGFR-sensitive clones are constrained and instead SCLC clones expand and result in a majority SCLC histological subtype. (II) Hypothesis of tumor stem cell: tumor stem cells with sensitive mutation 
Table 2 The treatment methods of 10 patients with lung adenocarcinoma before SCLC transformation

\begin{tabular}{|c|c|c|c|c|c|}
\hline Case No. & Surgery & Targeted therapy & Chemotherapy & Radiotherapy & Bisphosphonate \\
\hline 1 & $\Delta[1]$ & $\boldsymbol{\Delta}[3]$ & $\boldsymbol{\Delta}$ [2] & $\triangle$ & $\triangle$ \\
\hline 2 & $\Delta[1]$ & $\boldsymbol{\Delta}[3]$ & $\boldsymbol{\Delta}[2]$ & $\boldsymbol{\Delta}[3]$ & $\triangle$ \\
\hline 3 & $\triangle$ & $\boldsymbol{\Delta}[1.2]$ & $\triangle$ & $\triangle$ & $\triangle$ \\
\hline 4 & $\triangle$ & $\Delta[1]$ & $\boldsymbol{\Delta}[2]$ & $\Delta[1]$ & $\Delta[1]$ \\
\hline 5 & $\triangle$ & $\Delta[1.2 .3]$ & $\Delta[4]$ & $\triangle$ & $\triangle$ \\
\hline 6 & $\triangle$ & $\boldsymbol{\Delta}[1.2]$ & $\boldsymbol{\Delta}[1]$ & $\boldsymbol{\Delta}[2]$ & $\Delta[1]$ \\
\hline 7 & $\triangle$ & $\Delta[1.3 .4]$ & $\boldsymbol{\Delta}[2.6]$ & $\Delta[5]$ & $\triangle$ \\
\hline 8 & $\boldsymbol{\Delta}[1.3]$ & $\Delta[5]$ & $\boldsymbol{\Delta}[2]$ & $\Delta[4]$ & $\boldsymbol{\Delta}[2]$ \\
\hline 9 & $\triangle$ & $\Delta[1]$ & $\Delta[1]$ & $\boldsymbol{\Delta}[1]$ & $\triangle$ \\
\hline 10 & $\triangle$ & $\triangle$ & $\boldsymbol{\Delta}[1.2]$ & $\boldsymbol{\Delta}[2]$ & $\triangle$ \\
\hline
\end{tabular}

$\Delta$ means yes, $\triangle$ means no, the numbers in [ ] are the order of treatment plan. SCLC, small cell lung cancer.
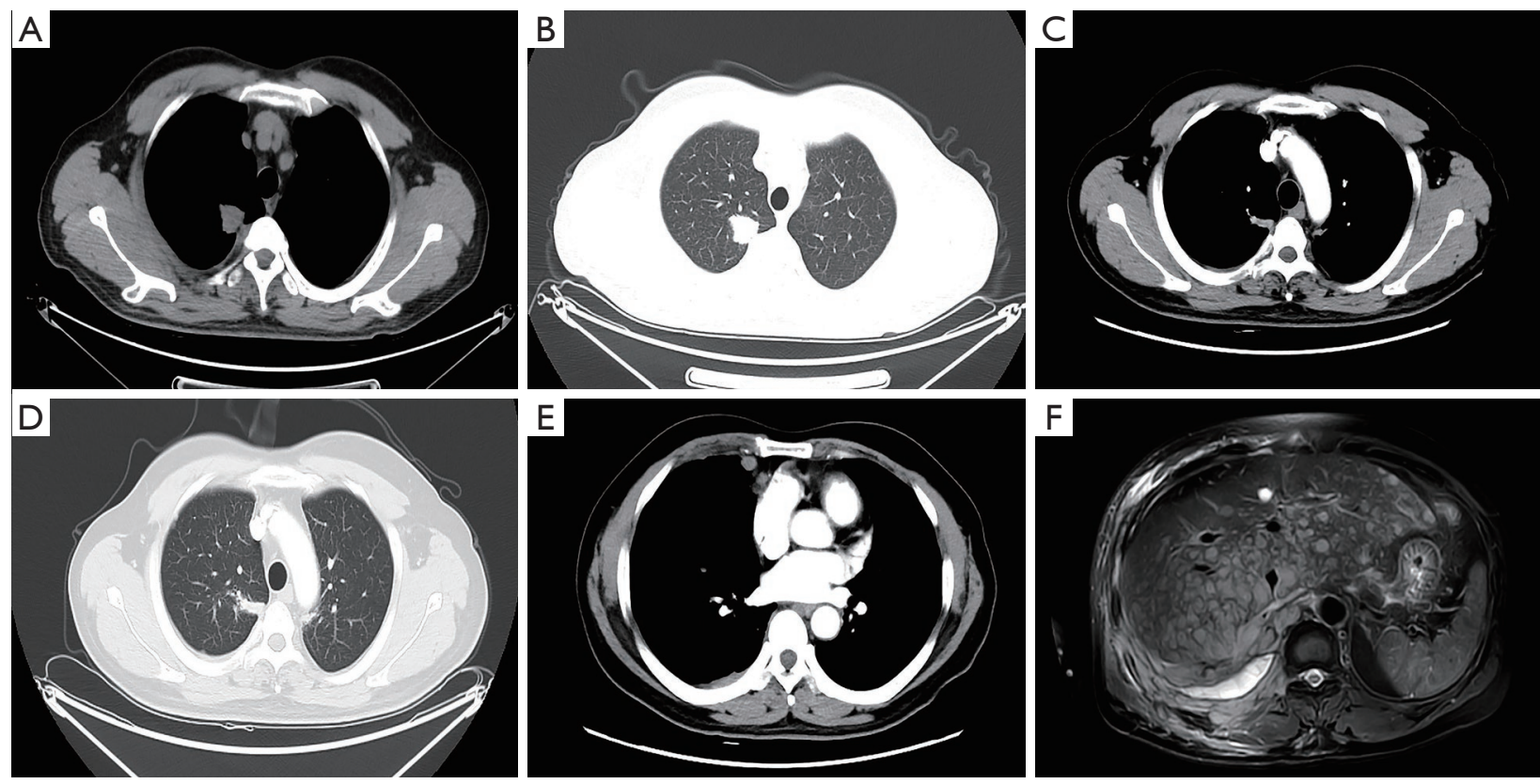

Figure 2 Images of case 4 at initial diagnosis and after transformation. (A) Chest CT in mediastinal window at initial diagnosis (adenocarcinoma). (B) Chest CT in pulmonary window at initial diagnosis (adenocarcinoma). (C) Chest CT in mediastinal window after transformation (SCLC). (D) Chest CT in pulmonary window after transformation (SCLC). (E) CT manifestations of pleural metastases after transformation (SCLC). (F) MRI manifestations of liver metastases after transformation (SCLC). CT, computed tomography; SCLC, small cell lung cancer; MRI, magnetic resonance imaging.

have the potential to differentiate into neuroendocrine tumor cells, EGFR TKIs select for EGFR-resistant clones in the adenocarcinoma that then subsequently transform into SCLC. (III) Hypothesis of molecular mechanism: during the treatment of TKIs, dual deletion mutations of tumor suppressor genes RB1 and TP53 appear, and they play an important role in SCLC transformation. Nowadays, more and more researchers are inclined to the second view. Sequist et al. performed second biopsies on patients diagnosed with lung adenocarcinoma with $E G F R$ 
Table 3 The expression of pathological immunohistochemical indicators after SCLC transformation

\begin{tabular}{|c|c|c|c|c|c|c|c|c|}
\hline Case No. & TTF1 & Napsin A & Syn & CD56 & Ki67 & $\mathrm{CgA}$ & AE1/AE3 & PD-L1 \\
\hline 2 & $(+)$ & $(-)$ & $(+)$ & $(+)$ & $90 \%+$ & $(+/-)$ & $(+)$ & $(-)$ \\
\hline 3 & $(+)$ & $(-)$ & $(+)$ & $(+)$ & $70 \%+$ & $(-)$ & $(+)$ & $(-)$ \\
\hline 4 & $(+)$ & $(-)$ & $(+)$ & $(+)$ & $80 \%+$ & $(-)$ & $(+)$ & $(-)$ \\
\hline 6 & $(+)$ & $(-)$ & $(+)$ & $(+)$ & $80 \%+$ & $(+)$ & $(+)$ & $(-)$ \\
\hline 7 & $(+)$ & $(-)$ & $(+)$ & $(+)$ & $90 \%+$ & $(+)$ & $(+)$ & $(-)$ \\
\hline 8 & $(+)$ & $(-)$ & $(+)$ & $(+)$ & $80 \%+$ & $(+)$ & $(+)$ & $(-)$ \\
\hline 9 & $(+)$ & $(-)$ & $(+)$ & $(+)$ & $70 \%+$ & $(-)$ & $(+)$ & $(-)$ \\
\hline
\end{tabular}

“+” means positive; “-" means negative. TTF1, thyroid transcription factor 1; Syn, synaptophysin; CgA, chromogranin A; SCLC, small cell lung cancer; PD-L1, programmed cell death-ligand 1.
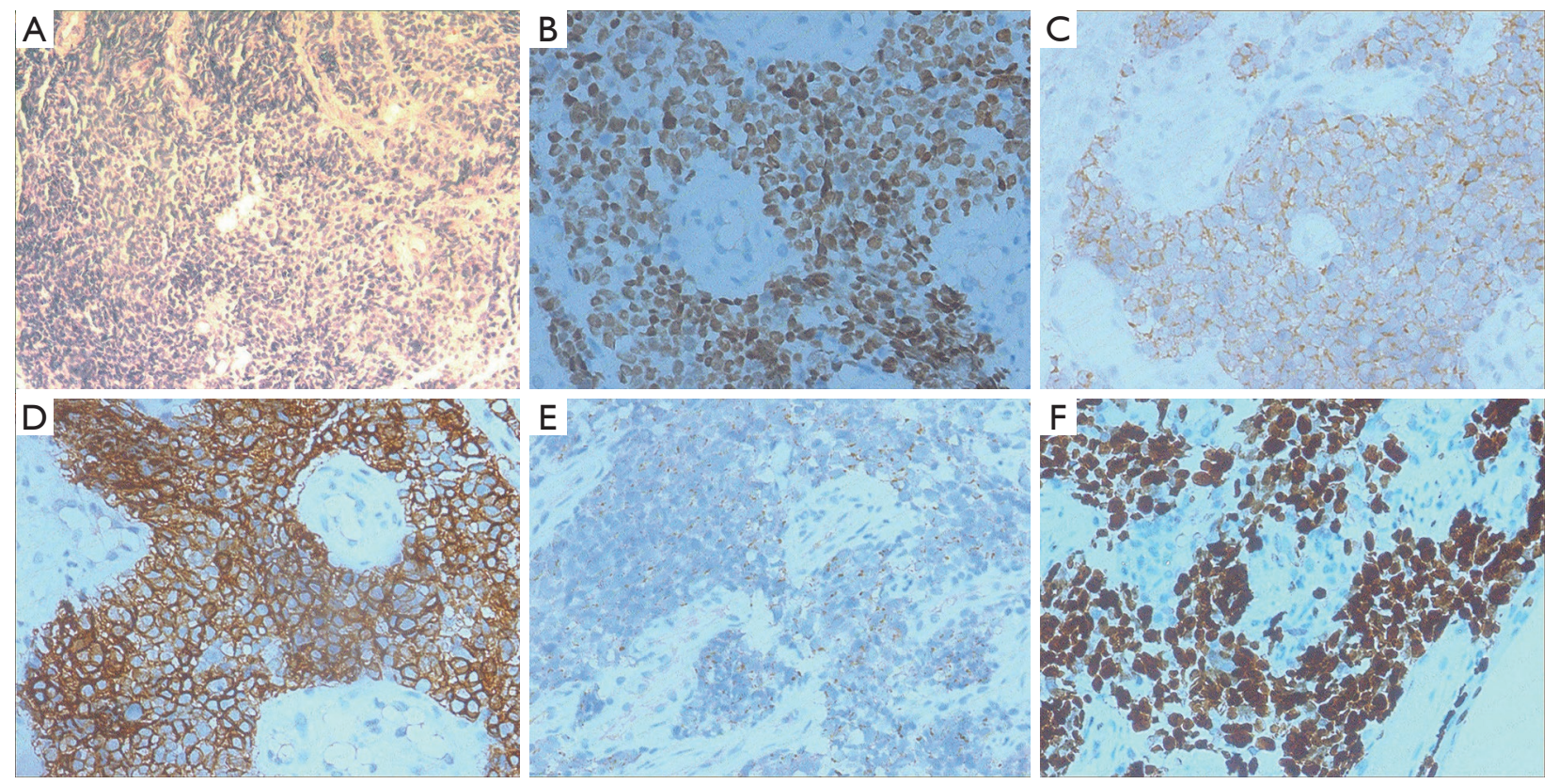

Figure 3 Pathological and immunohistochemical examination of case 1 after transformation (SCLC). (A) Pathology after transformation (H\&E, $\times 10$ ). (B) TTF-1 positive (IHC, $\times 20$ ). (C) Syn positive (IHC, $\times 20)$. (D) CD56 positive (IHC, $\times 20)$. (E) CgA positive (IHC, ×20). (F) Ki67 high expression (IHC, $\times 20$ ). H\&E, hematoxylin-eosin staining; IHC, immunohistochemical staining; TTF-1, thyroid transcription factor 1; Syn, synaptophysin; CD56, nerve cell adhesion molecule CD56; CgA, chromogranin A; Ki67, Ki67 antigen.

gene mutation and resistance to targeted therapy (8). The results showed that the pathological types have transformed into SCLC, and the transformed SCLC tissues still maintained the original EGFR mutation type, suggesting that it may have originated from the same consistency tissues. Shi et al. showed that patients generally possessed consistent types of EGFR mutation before and after transformation, indicating that SCLC after TKI treatment originated from adenocarcinoma (9). Our findings that 8 of 10 patients who maintained the original EGFR gene 
Table 4 The transformation interval of different treatment sequence groups

\begin{tabular}{lcc}
\hline The therapeutic sequence before transformation & Case & The time interval of transformation [mean] (months) \\
\hline $\mathrm{C}+\mathrm{T}$ & $1,2,8$ & $38,40,31[36]$ \\
$\mathrm{T}+\mathrm{C}$ & $4,5,7$ & $13,14,40[22]$ \\
$\mathrm{CCT}$ & 6,9 & $15,7[11]$ \\
\hline
\end{tabular}

$\mathrm{C}+\mathrm{T}$, chemotherapy followed by targeted therapy; $\mathrm{T}+\mathrm{C}$, targeted therapy followed by chemotherapy; CCT, concurrent chemotherapy combined with targeted therapy.

Table 5 Time to SCLC transformation, treatment, and outcome of 10 patients after SCLC diagnosis

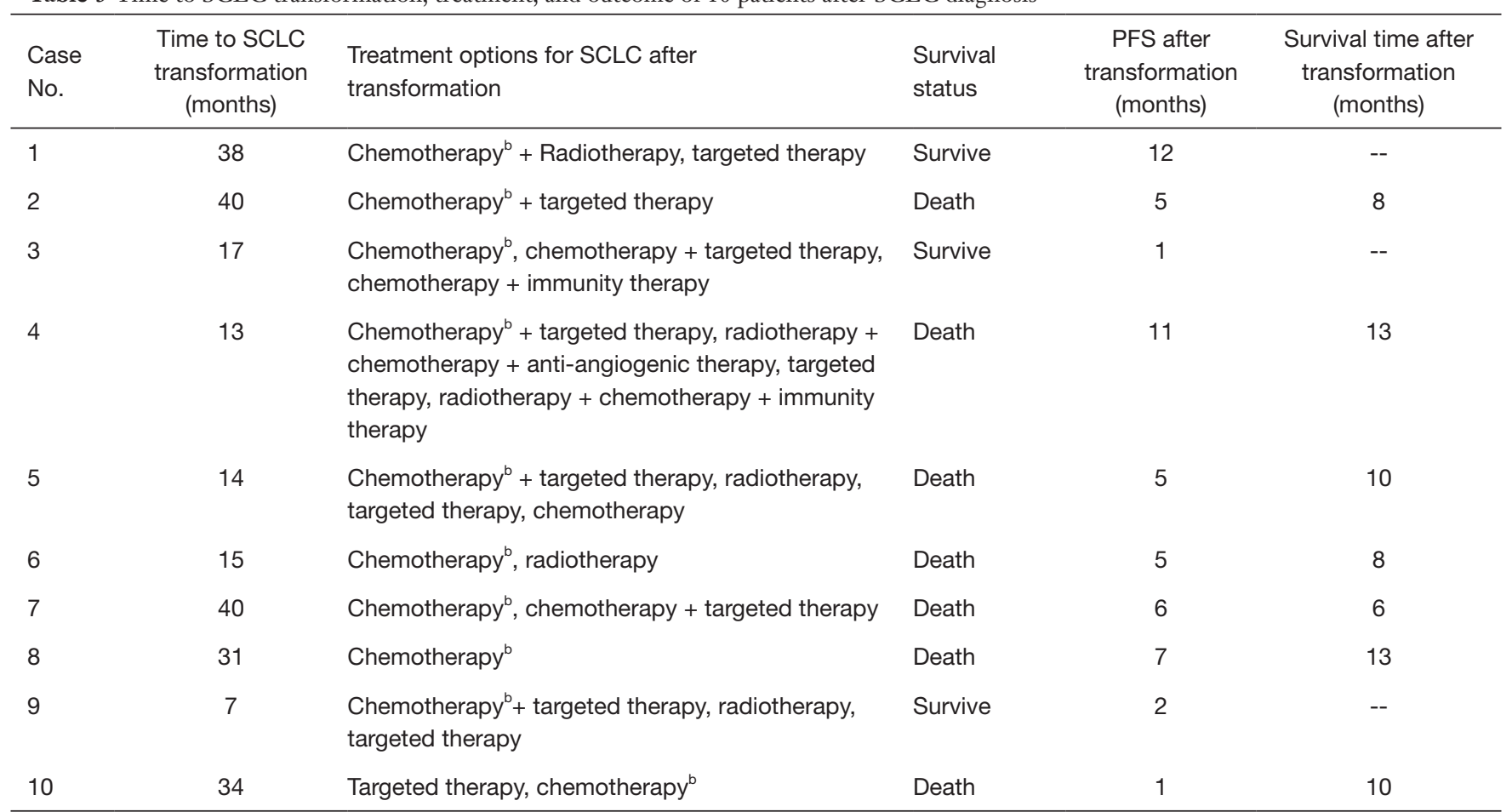

${ }^{\mathrm{b}}$, the regimen of EP (etoposide plus cisplatin) is the first choice for post-transformation chemotherapy. "--" means that the patient is alive. SCLC, small cell lung cancer.

mutation after transformation are largely compatible with the two aforementioned studies. Although case 10 presented an EGFR E19 mutation at the second examination, this difference may be attributed to inconsistency in the testing subjects as the specimens for genetic testing were blood and tissue before and after transformation, respectively.

Marcoux et al. retrospectively reviewed 67 patients with EGFR mutant SCLC and other high-grade neuroendocrine carcinomas (10). Among them, EGFR mutations included exon 19 deletion (69\%), L858R (25\%), and others (6\%). At the initial lung cancer diagnosis, 58 patients had NSCLC and nine had de novo SCLC or mixed histology. All these nine patients were treated with one or more TKIs prior to transformation. After transformation, both platinum etoposide and taxanes yielded high response rates, whereas the median PFS was only 3.4 months (2.4 to 5.4 months), and the median survival was only 10.9 months (8.0 to 13.7 months). The results of Ahmed et al. showed that the median time to transformation was 36 months in 30 patients with lung adenocarcinoma who received multiple lines of therapy (11). Besides, Roca et al. reported that the median time from initial diagnosis of lung adenocarcinoma to SCLC transformation was 19 months, and the median survival time after SCLC diagnosis was 
6 months (12). Furthermore, Ferrer et al. found that patients with EGFR mutant NSCLC were more likely to develop SCLC transformation after multi lines of therapy, and that the majority retained a consistent mutation type (13). Our results are similar to those of the mentioned studies and suggest that aggressive second biopsy and genetic testing for patients with lung adenocarcinoma whose diseases progress after resistance to EGFR-TKI therapy or multiple lines of therapy are clinically warranted to select appropriate therapeutic strategies.

None of the 10 patients in this study was older than 70 years, with a mean age of 55 years. Studies have shown that elderly patients ( $\geq 65$ years) with advanced NSCLC harboring EGFR sensitive mutations demonstrated longer PFS after EGFR-TKI treatment than younger patients $(14,15)$, which may be attributed to the slow metabolism and corresponding alleviated tumor progression in the elderly. In recent years, many studies have shown that EGFR-TKIs in combination with chemotherapy can delay drug resistance and prolong PFS and OS in patients with EGFR mutation-positive advanced NSCLC (16-19). Some researchers have reported a correlation between gene expression and sensitivity to cancer agents in cell line models $(20,21)$. In addition, patients with lung adenocarcinoma who received chemotherapy followed by targeted therapy exhibited a longer time interval of transformation than patients who received targeted therapy first. These findings suggest that perhaps chemotherapy delays the alteration of resistance related genes, but the mechanism requires further investigation.

NSE, a highly specific and sensitive tumor marker, plays a critical role in the diagnosis of SCLC (22). We observed an increase in NSE level in this study, indicating that NSE can be considered as a detection indicator. If patients' NSE increased with short-term and multi-site progression, attention should be paid to the possibility of SCLC transformation. The chemotherapy regimen of EC (etoposide plus carboplatin) or EP (etoposide plus cisplatin) in combination with immunosuppressive therapy has become the first choice for extensive staging SCLC $(23,24)$. PD-L1 was not expressed in ten patients in this study, and two of them failed to undergo immunotherapy in subsequent treatments after transformation. In the study by Marcoux et al., immune checkpoint inhibitors were ineffective in 17 patients (10), indicating that metrics to screen specific populations suitable for immunotherapy after transformation require further exploration.

There are certain limitations to this study. We did not test the RB1 and TP53 status in any of the patients at the first visit. For lung adenocarcinoma patients treated with EGFR-TKI, evaluating the RB1 and TP53 status is of great significance for predicting the occurrence and prognosis of SCLC transformation. Lee et al. in 2017 found a 42.8-fold increased relative risk of EGFR mutant lung adenocarcinoma with $R B 1$ and TP53 inactivation at first diagnosis (25). In 2019, Offin et al. at the Sloan Kettering Cancer Center analyzed 43 patients with EGFR/TP53/ $R B 1$ triple mutations, including 7 (18\%) patients with lung adenocarcinoma who developed SCLC transformation during treatment (26). The median time from initial TKI treatment to transformation was 1.1 years, whereas patients with triple mutations had even shorter intervals.

As biopsy technology advances and genetic testing technology matures, more and more cases of SCLC transformation after lung adenocarcinoma treatment will be identified. In clinical work, awareness of secondary biopsies should be increased and whole-genome sequencing could be performed depending on the severity of the diseases, in hopes of earlier detection of SCLC transformation and development of a precise treatment strategy.

\section{Acknowledgments}

The authors would like to thank the patients and their families who participated in this study, and the colleagues from the Fourth Hospital of Hebei Medical University for their contribution to the study. The authors also acknowledge TopEdit LLC for the linguistic editing and proofreading during the preparation of this manuscript.

Funding: This work was supported by the tracking project of medical applicable technology in Hebei Province, China (grant number G2019078 to Z Zhou).

\section{Footnote}

Reporting Checklist: The authors have completed the STROBE reporting checklist. Available at https://dx.doi. org/10.21037/tcr-21-653

Data Sharing Statement: Available at https://dx.doi. org/10.21037/tcr-21-653

Peer Review File: Available at https://dx.doi.org/10.21037/ tcr-21-653

Conflicts of Interest: All authors have completed the ICMJE 
uniform disclosure form (available at https://dx.doi. org/10.21037/tcr-21-653). The authors have no conflicts of interest to declare.

Ethical Statement: The authors are accountable for all aspects of the work in ensuring that questions related to the accuracy or integrity of any part of the work are appropriately investigated and resolved. The study was conducted in accordance with the Declaration of Helsinki (as revised in 2013). The study was approved by the Ethics Committee board of the Fourth Hospital of Hebei Medical University (2020KY254) and individual consent for this retrospective analysis was waived.

Open Access Statement: This is an Open Access article distributed in accordance with the Creative Commons Attribution-NonCommercial-NoDerivs 4.0 International License (CC BY-NC-ND 4.0), which permits the noncommercial replication and distribution of the article with the strict proviso that no changes or edits are made and the original work is properly cited (including links to both the formal publication through the relevant DOI and the license). See: https://creativecommons.org/licenses/by-nc-nd/4.0/.

\section{References}

1. Bray F, Ferlay J, Soerjomataram I, et al. Global cancer statistics 2018: GLOBOCAN estimates of incidence and mortality worldwide for 36 cancers in 185 countries. CA Cancer J Clin 2018;68:394-424.

2. Engelman JA, Jänne PA. Mechanisms of acquired resistance to epidermal growth factor receptor tyrosine kinase inhibitors in non-small cell lung cancer. Clin Cancer Res 2008;14:2895-9.

3. Lim SM, Syn NL, Cho BC, et al. Acquired resistance to EGFR targeted therapy in non-small cell lung cancer: Mechanisms and therapeutic strategies. Cancer Treat Rev 2018;65:1-10.

4. Zakowski MF, Ladanyi M, Kris MG, et al. EGFR mutations in small-cell lung cancers in patients who have never smoked. N Engl J Med 2006;355:213-5.

5. Watanabe S, Sone T, Matsui T, et al. Transformation to small-cell lung cancer following treatment with EGFR tyrosine kinase inhibitors in a patient with lung adenocarcinoma. Lung Cancer 2013;82:370-2.

6. Ahn S, Hwang SH, Han J, et al. Transformation to Small Cell Lung Cancer of Pulmonary Adenocarcinoma: Clinicopathologic Analysis of Six Cases. J Pathol Transl
Med 2016;50:258-63.

7. Ahmed T, Vial MR, Ost D, et al. Non-small cell lung cancer transdifferentiation into small cell lung cancer: A case series. Lung Cancer 2018;122:220-3.

8. Sequist LV, Waltman BA, Dias-Santagata D, et al. Genotypic and histological evolution of lung cancers acquiring resistance to EGFR inhibitors. Sci Transl Med 2011;3:75ra26.

9. Shi X, Duan H, Liu X, et al. Genetic alterations and protein expression in combined small cell lung cancers and small cell lung cancers arising from lung adenocarcinomas after therapy with tyrosine kinase inhibitors. Oncotarget 2016;7:34240-9.

10. Marcoux N, Gettinger SN, O'Kane G, et al. EGFRMutant Adenocarcinomas That Transform to Small-Cell Lung Cancer and Other Neuroendocrine Carcinomas: Clinical Outcomes. J Clin Oncol 2019;37:278-85.

11. Ahmed T, Vial MR, Ost D, et al. Non-small cell lung cancer transdifferentiation into small cell lung cancer: A case series. Lung Cancer 2018;122:220-3.

12. Roca E, Gurizzan C, Amoroso V, et al. Outcome of patients with lung adenocarcinoma with transformation to small-cell lung cancer following tyrosine kinase inhibitors treatment: A systematic review and pooled analysis. Cancer Treat Rev 2017;59:117-22.

13. Ferrer L, Giaj Levra M, Brevet M, et al. A Brief Report of Transformation From NSCLC to SCLC: Molecular and Therapeutic Characteristics. J Thorac Oncol 2019;14:130-4.

14. Rosell R, Carcereny E, Gervais R, et al. Erlotinib versus standard chemotherapy as first-line treatment for European patients with advanced EGFR mutation-positive non-small-cell lung cancer (EURTAC): a multicentre, open-label, randomised phase 3 trial. Lancet Oncol 2012;13:239-46.

15. Wu YL, Zhou C, Hu CP, et al. Afatinib versus cisplatin plus gemcitabine for first-line treatment of Asian patients with advanced non-small-cell lung cancer harbouring EGFR mutations (LUX-Lung 6): an open-label, randomised phase 3 trial. Lancet Oncol 2014;15:213-22.

16. Hosomi Y, Morita S, Sugawara S, et al. Gefitinib Alone Versus Gefitinib Plus Chemotherapy for Non-Small-Cell Lung Cancer With Mutated Epidermal Growth Factor Receptor: NEJ009 Study. J Clin Oncol 2020;38:115-23.

17. Noronha V, Patil VM, Joshi A, et al. Gefitinib Versus Gefitinib Plus Pemetrexed and Carboplatin Chemotherapy in EGFR-Mutated Lung Cancer. J Clin Oncol 2020;38:124-36. 
18. Wen M, Xia J, Sun Y, et al. Combination of EGFR-TKIs with chemotherapy versus chemotherapy or EGFR-TKIs alone in advanced NSCLC patients with EGFR mutation. Biologics 2018;12:183-90.

19. Han B, Jin B, Chu T, et al. Combination of chemotherapy and gefitinib as first-line treatment for patients with advanced lung adenocarcinoma and sensitive EGFR mutations: A randomized controlled trial. Int J Cancer 2017;141:1249-56.

20. Vural S, Simon R, Krushkal J. Correlation of gene expression and associated mutation profiles of APOBEC3A, APOBEC3B, REV1, UNG, and FHIT with chemosensitivity of cancer cell lines to drug treatment. Hum Genomics 2018;12:20.

21. Pich $\mathrm{O}$, Muiños F, Lolkema MP, et al. The mutational footprints of cancer therapies. Nat Genet 2019;51:1732-40.

22. Kulpa J, Wójcik E, Reinfuss M, et al. Carcinoembryonic antigen, squamous cell carcinoma antigen, CYFRA 21-1,

Cite this article as: Bai W, Zhen C, Zhang R, Yu W, Zhou Z. Clinicopathological features of patients with transformation from EGFR mutant lung adenocarcinoma to small cell lung cancer. Transl Cancer Res 2021;10(8):3694-3704. doi: 10.21037/ tcr-21-653 and neuron-specific enolase in squamous cell lung cancer patients. Clin Chem 2002;48:1931-7.

23. Horn L, Mansfield AS, Szczęsna A, et al. First-Line Atezolizumab plus Chemotherapy in Extensive-Stage Small-Cell Lung Cancer. N Engl J Med 2018;379:2220-9.

24. Paz-Ares L, Dvorkin M, Chen Y, et al. Durvalumab plus platinum-etoposide versus platinum-etoposide in firstline treatment of extensive-stage small-cell lung cancer (CASPIAN): a randomised, controlled, open-label, phase 3 trial. Lancet 2019;394:1929-39.

25. Lee JK, Lee J, Kim S, et al. Clonal History and Genetic Predictors of Transformation Into Small-Cell Carcinomas From Lung Adenocarcinomas. J Clin Oncol 2017;35:3065-74.

26. Offin M, Chan JM, Tenet $M$, et al. Concurrent RB1 and TP53 Alterations Define a Subset of EGFR-Mutant Lung Cancers at risk for Histologic Transformation and Inferior Clinical Outcomes. J Thorac Oncol 2019;14:1784-93. 